\title{
A ROLLE TYPE THEOREM FOR CYCLICITY OF ZEROS OF FAMILIES OF ANALYTIC FUNCTIONS
}

\author{
ALEXANDER BRUDNYI
}

\begin{abstract}
Let $\left\{f_{\lambda ; j}\right\}_{\lambda \in V ; 1 \leq j \leq k}$ be families of holomorphic functions in the open unit disk $\mathbb{D} \subset \mathbb{C}$ depending holomorphically on a parameter $\lambda \in V \subset \mathbb{C}^{n}$. We establish a Rolle type theorem for the generalized multiplicity (called cyclicity) of zero of the family of univariate holomorphic functions $\left\{\sum_{j=1}^{k} f_{\lambda ; j}\right\}_{\lambda \in V}$ at $0 \in \mathbb{D}$. As a corollary, we estimate the cyclicity of the family of generalized exponential polynomials, that is, the family of entire functions of the form $\sum_{k=1}^{m} P_{k}(z) e^{Q_{k}(z)}, z \in \mathbb{C}$, where $P_{k}$ and $Q_{k}$ are holomorphic polynomials of degrees $p$ and $q$, respectively, parameterized by vectors of coefficients of $P_{k}$ and $Q_{k}$.
\end{abstract}

\section{INTRODUCTION}

1.1. Basic Definitions and Results. Let $B_{n}(\lambda, r) \subset \mathbb{C}^{n}$ be the open Euclidean ball centered at $\lambda$ of radius $r$. We set $\mathbb{D}_{r}:=B_{1}(0, r) \subset \mathbb{C}$ and $\mathbb{D}:=\mathbb{D}_{1}$. By $K_{\varepsilon}:=\cup_{\lambda \in K} B_{n}(\lambda, \varepsilon)$ we denote the $\varepsilon$-neighbourhood of $K \subset \mathbb{C}^{n}$. Let $V \subset \mathbb{C}^{n}$ be a domain and $\mathcal{O}(V)$ be the ring of holomorphic functions on $V$. Consider the Maclaurin series expansion of the family $\mathcal{F}=\left\{f_{\lambda}\right\}_{\lambda \in V}$ of holomorphic functions in the disk $\mathbb{D}_{\rho}$ depending holomorphically on $\lambda \in V$,

$$
f_{\lambda}(z)=\sum_{k=0}^{\infty} a_{k}(\lambda) z^{k}, \quad \lambda \in V, \quad z \in \mathbb{D}_{\rho}, \quad a_{k} \in \mathcal{O}(V) .
$$

Let $U \subset V$ be a subdomain. By $\mathcal{I}(\mathcal{F} ; U) \subset \mathcal{O}(U)$ we denote the ideal generated by all the restrictions $\left.a_{k}\right|_{U}$. The central set of $\mathcal{F}$ in $U$ is defined by the formula

$$
\mathcal{C}(\mathcal{F} ; U):=\left\{\lambda \in U: f_{\lambda} \equiv 0\right\} .
$$

Definition 1.1. The family $\mathcal{F}=\left\{f_{\lambda}\right\}_{\lambda \in V}$ has cyclicity $k \geq 0$ in a compact set $K \subset V$ if the following holds:

(a) There exist $\varepsilon_{0}>0$ and $\delta_{0}>0$ such that for every $\lambda$ in $K_{\varepsilon_{0}} \cap(V \backslash \mathcal{C}(\mathcal{F} ; V))$ the function $f_{\lambda}$ has at most $k$ zeros in $\mathbb{D}_{\delta_{0}}$ (counting multiplicities).

(b) For arbitrary positive numbers $\varepsilon<\varepsilon_{0}$ and $\delta<\delta_{0}$ there exists a parameter $\lambda \in$ $K_{\varepsilon} \cap(V \backslash \mathcal{C}(\mathcal{F} ; V))$ such that $f_{\lambda}$ has exactly $k$ zeros in $\mathbb{D}_{\delta}$.

The notion goes back to the famous paper [B1] (see also [B2]) of Bautin who found a connection between cyclicity and algebraic properties of $\mathcal{I}(\mathcal{F} ; V)$ and in this way proved that a perturbation of a center in a family of quadratic vector fields can generate no more than 3 small amplitude limit cycles.

The cyclicity of the family $\mathcal{F}$ in $K$ is denoted by $c(\mathcal{F} ; K)$. For a one point compact set $K:=\{\mu\}$ we use the notation $c(\mathcal{F} ; \mu)$. For $\mu \notin \mathcal{C}(\mathcal{F} ; V)$ this number equals the multiplicity of the zero of $f_{\mu}$ at $0 \in \mathbb{D}$. Therefore the term "generalized multiplicity" seems to be quite natural for $c(\mathcal{F} ; K)$.

2010 Mathematics Subject Classification. Primary 30C15, Secondary 32A15.

Key words and phrases. Cyclicity of zero, holomorphic function, Wronskian determinant, Cartan estimates, Remez inequality.

Research supported in part by NSERC. 
The problem of bounding cyclicity for specific families $\mathcal{F}$ arises in several diverse areas of analysis, including dynamical systems (related to the, so-called, second part of Hilbert's 16 problem), transcendental number theory (related to Hilbert's 7 problem) and approximation theory (Bernstein-Markov-Remez' type inequalities); for recent developments and corresponding references, see, e.g., $\mathrm{Y}$ ] and the Featured Review [MR 98h:34009].

The basic properties of cyclicity are obtained in Br1]. We formulate some of them.

Let $\mathcal{F}=\left\{f_{\lambda}\right\}$ and $\mathcal{G}=\left\{g_{\lambda}\right\}$ be families of holomorphic functions in $\mathbb{D}_{\rho}$ depending holomorphically on $\lambda \in V$. We set

$$
\mathcal{F}^{\prime}:=\left\{\frac{d f_{\lambda}}{d z}\right\}, \quad \mathcal{F}+\mathcal{G}:=\left\{f_{\lambda}+g_{\lambda}\right\}, \quad \mathcal{F} \cdot \mathcal{G}:=\left\{f_{\lambda} \cdot g_{\lambda}\right\} \quad \text { and } \quad e^{\mathcal{F}}:=\left\{e^{f_{\lambda}}\right\} .
$$

The following properties are presented in Propositions 2.1, 2.2, 2.5, Theorems 1.5, 1.7 and Remark 1.6 of [Br1] (see also this paper for additional references).

(a) For a compact set $K \subset V$,

$$
c(\mathcal{F} ; K)=\max _{\mu \in K} c(\mathcal{F} ; \mu) .
$$

(b) There exist a constant $A$ and an open neighbourhood $U$ of $K$ such that (see (1.1))

$$
\left|a_{k}(\lambda)\right| \leq A \cdot \sup _{U}\left|a_{k}\right| \cdot \max _{0 \leq i \leq c(\mathcal{F} ; K)}\left|a_{i}(\lambda)\right| \quad \text { for all } \quad \lambda \in U ;
$$

here $c(\mathcal{F} ; K)$ cannot be replaced by a smaller index.

If $K=\{\mu\}$, the latter property is equivalent to the following one (see [HRT]).

Let $\mathcal{I}(\mathcal{F} ; \mu)$ be the ideal in the local ring $\mathcal{O}_{\mu}$ of germs of holomorphic functions at $\mu \in V$ generated by germs at $\mu$ of the Taylor coefficients $a_{j}$ of $\mathcal{F}$, see (1.1), and $\mathcal{I}_{d}(\mathcal{F} ; \mu) \subset \mathcal{I}(\mathcal{F} ; \mu)$ be the ideal generated by germs at $\mu$ of $a_{0}, \ldots, a_{d}$. We define $d(\mathcal{F} ; \mu)$ to be the minimal integer $d$ such that the integral closures of $\mathcal{I}_{d}(\mathcal{F} ; \mu)$ and $\mathcal{I}(\mathcal{F} ; \mu)$ coincide.

$\left(b^{\prime}\right)$

$$
c(\mathcal{F} ; \mu)=d(\mathcal{F} ; \mu) .
$$

(c) For a compact set $K \subset V$ there exists an open neighbourhood $O_{K}$ of $K$ such that the central set $\mathcal{C}\left(\mathcal{F} ; O_{K}\right)$ coincides with the set of common zeros of $a_{0}, \ldots, a_{c(\mathcal{F} ; K)}$.

(e)

$$
c(\mathcal{F} ; K) \leq c\left(\mathcal{F}^{\prime} ; K\right)+1
$$

$$
c\left(e^{\mathcal{F}} ; K\right)=0
$$

$$
c(\mathcal{F} \cdot \mathcal{G} ; K) \leq c(\mathcal{F} ; K)+c(\mathcal{G} ; K)
$$

$$
c(\mathcal{F}+\mathcal{G} ; K) \leq \max \{c(\mathcal{F} ; K), c(\mathcal{G} ; K), c(W(\mathcal{F}, \mathcal{G}) ; K)+1\}
$$

here $W(\mathcal{F}, \mathcal{G}):=\mathcal{F}^{\prime} \cdot \mathcal{G}-\mathcal{G}^{\prime} \cdot \mathcal{F}$ is the family of Wronskian determinants of $\{\mathcal{F}, \mathcal{G}\}$.

(h) For every $\varepsilon>0$ there exist an open neighbourhood $O_{\varepsilon}$ of $K$ and a positive number $R_{\varepsilon}$ such that for all $\lambda \in O_{\varepsilon} \backslash \mathcal{C}(\mathcal{F} ; V)$

$$
\sup _{\mathbb{D}_{R_{\varepsilon}}} \ln \left|f_{\lambda}\right|-\sup _{\mathbb{D}_{R_{\varepsilon} / e}} \ln \left|f_{\lambda}\right| \leq c(\mathcal{F} ; K)+\varepsilon
$$

and each $f_{\lambda}$ has at most $c(\mathcal{F} ; K)$ zeros in $\mathbb{D}_{R_{\varepsilon}}$ (counting multiplicities); here $c(\mathcal{F} ; K)$ cannot be replaced by a smaller number.

Property (h) generates the following Cartan-type estimates and Remez-type inequalities for the family $\mathcal{F}$, see, e.g., [Br2, Th. 3.1 and Sect. 2] and references therein. 
$\left(\mathrm{h}^{\prime}\right)$ Fix $H \in(0,1], d>0$ and set $A:=e^{\left(\frac{e+1}{e-1}\right)^{2}}$. For each $\lambda \in O_{\varepsilon} \backslash \mathcal{C}(\mathcal{F} ; V)$ and $R \in\left(0, R_{\varepsilon}\right)$ there exists a family of open disks $\left\{D_{j ; \lambda}\right\}_{1 \leq j \leq k}, k \leq c(\mathcal{F} ; K)$, with $\sum r_{j ; \lambda}^{d} \leq \frac{(2 H R)^{d}}{d}$, where $r_{j ; \lambda}$ is the radius of $D_{j ; \lambda}$, such that

$$
\left|f_{\lambda}(z)\right| \geq \sup _{\mathbb{D}_{R}}\left|f_{\lambda}\right| \cdot A^{-\varepsilon} \cdot\left(\frac{H}{A}\right)^{c(\mathcal{F} ; K)} \quad \text { for all } \quad z \in \mathbb{D}_{R / e} \backslash \cup_{j} D_{j ; \lambda} .
$$

$\left(\mathrm{h}^{\prime \prime}\right)$ Consider the function $\Phi(t):=t+\sqrt{t^{2}-1}, t \geq 1$. There exists an absolute constant $\hat{c} \geq 1$ such that for each $\lambda \in O_{1}$, an interval $I \subset \mathbb{D}_{R_{1} / e}$ and a measurable subset $\omega \subset I$ the following inequality holds:

$$
\sup _{I}\left|f_{\lambda}\right| \leq \Phi\left(\frac{2 m_{1}(I)}{m_{1}(\omega)}-1\right)^{\hat{c} \cdot \max \{c(\mathcal{F} ; K), 1\}} \cdot \sup _{\omega}\left|f_{\lambda}\right| ;
$$

here $m_{1}$ is the linear measure on $\mathbb{C}$.

1.2. Formulation of the Main Result. In this note we generalize property (g) and establish a Role type theorem for the cyclicity of the family $\mathcal{F}:=\sum_{j=1}^{k} \mathcal{F}_{j}$, where $\mathcal{F}_{j}:=$ $\left\{f_{\lambda ; j}\right\}, 1 \leq j \leq k$, are families of holomorphic functions in $\mathbb{D}_{\rho} \subset \mathbb{C}$ holomorphic in $\lambda \in V \subset \mathbb{C}^{n}$. As a corollary, in the next section we estimate the cyclicity of the family of generalized exponential polynomials.

In our main result $W\left(\mathcal{F}_{i_{1}}, \ldots, \mathcal{F}_{i_{l}}\right):=\left\{W\left(f_{\lambda ; i_{1}}, \ldots, f_{\lambda ; i_{l}}\right)\right\}_{\lambda \in V}, 1 \leq i_{1}<\cdots<i_{l} \leq k$, stands for the family of Wronskian determinants

$$
W\left(f_{\lambda ; i_{1}}, \ldots, f_{\lambda ; i_{l}}\right):=\left|\begin{array}{cccc}
f_{\lambda ; i_{1}} & f_{\lambda ; i_{2}} & \ldots & f_{\lambda ; i_{l}} \\
f_{\lambda ; i_{1}}^{\prime} & f_{\lambda ; i_{2}}^{\prime} & \ldots & f_{\lambda ; i_{l}}^{\prime} \\
\vdots & \vdots & \vdots & \vdots \\
f_{\lambda ; i_{1}}^{(l-1)} & f_{\lambda ; i_{2}}^{(l-1)} & \ldots & f_{\lambda ; i_{l}}^{(l-1)}
\end{array}\right|
$$

Let $\mathcal{K}$ be the family of all distinct subsets of $\{1, \ldots, k\}$. By $|I|$ we denote the cardinality of $I \in \mathcal{K}$. For $I=\left\{i_{1}, \ldots, i_{l}\right\} \in \mathcal{K}$ and a compact subset $K \subset V$ we set

$$
c\left(\mathcal{F}_{I} ; K\right):=c\left(W\left(\mathcal{F}_{i_{1}}, \ldots, \mathcal{F}_{i_{l}}\right) ; K\right) .
$$

Theorem 1.2.

$$
c(\mathcal{F} ; K) \leq \max _{I \in \mathcal{K}}\left\{c\left(\mathcal{F}_{I} ; K\right)+|I|-1\right\} .
$$

For $K=\{\mu\} \subset V$ with $\mu \notin C(\mathcal{F} ; V)$ (i.e., in the case of the multiplicity of zero at $0 \in \mathbb{D}$ of $\left.f_{\mu} \not \equiv 0\right)$ this result was proved in [VP, Th. 1].

The proof of Theorem 1.2 is presented in Section 3.

\section{Cyclicity of the Family of Generalized Exponential Polynomials}

Consider the family $\mathcal{F}=\left\{f_{\lambda}\right\}$ of entire functions of the form

$$
\sum_{k=1}^{m} P_{k}(z) e^{Q_{k}(z)}, \quad z \in \mathbb{C},
$$

where $P_{k}(z)=\sum_{i=0}^{p} c_{k i} z^{i}$ and $Q_{k}(z)=\sum_{j=1}^{q} d_{k j} z^{j}$ are holomorphic polynomials of degrees $p$ and $q$, respectively. We parameterize elements of this family by strings $\lambda=$ $\left(\left(c_{k i}\right)_{0 \leq i \leq p},\left(d_{k j}\right)_{1 \leq j \leq q}\right)_{1 \leq k \leq m}$ of the coefficients of polynomials $P_{k}$ and $Q_{k}$ involved in their definition, i.e., by points of $\mathbb{C}^{N_{p, q, m}}$, where $N_{p, q, m}:=m(p+q+1)$. Then

$$
f_{\lambda}(z)=\sum_{n=0}^{\infty} a_{n}(\lambda) z^{n}, \quad z \in \mathbb{C},
$$


where $a_{n}$ is a holomorphic polynomial in $\lambda$ of degree $n+1$ with nonnegative rational coefficients. Using Taylor series expansion for the exponent together with the multinomial theorem we obtain

$$
a_{n}(\lambda)=\sum_{k=1}^{m}\left(\sum_{j=0}^{p} c_{k j}\left(\sum_{k_{1}+2 k_{2}+\cdots+q k_{q}=n-j} \frac{d_{k k_{1}}^{k_{1}} d_{k k_{2}}^{k_{2}} \cdots d_{k k_{q}}^{k_{q}}}{k_{1} ! k_{2} ! \cdots k_{q} !}\right)\right) .
$$

(Here the inner summation is taken over all sequences $k_{1}, \ldots, k_{q} \in \mathbb{Z}_{+}$satisfying the required condition.)

For instance,

$a_{0}(\lambda)=\sum_{k=1}^{m} c_{k 0}, \quad a_{1}(\lambda)=\sum_{k=1}^{m} c_{k 1}+c_{k 0} d_{k 1}, \quad a_{2}(\lambda)=\sum_{k=1}^{m} c_{k 2}+c_{k 1} d_{k 1}+c_{k 0}\left(d_{k 2}+\frac{d_{k 1}^{2}}{2}\right)$,

etc.

The center set $\mathcal{C}\left(\mathcal{F} ; \mathbb{C}^{N_{p, q, m}}\right)$ consists of points $\lambda \in \mathbb{C}^{N_{p, q, m}}$ for which the corresponding generalized exponential polynomials $f_{\lambda}$ are identically zero. Using a simple induction argument on $m$, based on the comparison of exponential and polynomial growth of entire functions, one obtains that $\sum_{k=1}^{m} P_{k} e^{Q_{k}} \equiv 0$ if and only if there exists a subset $I \subset$ $\{1, \ldots, m\}$ such that

(a) $Q_{i}=Q_{j}$ for all $i, j \in I$, and $Q_{i} \neq Q_{j}$ for all distinct $i, j \notin I$ or if $i \in I$ and $j \notin I$;

(b) $\sum_{k \in I} P_{k}=0$ and $P_{k}=0$ for all $k \notin I$.

Thus, $\mathcal{C}\left(\mathcal{F} ; \mathbb{C}^{N_{p, q, m}}\right)=\cup_{I} V_{I}$, where $I$ runs over all distinct subsets of $\{1, \ldots, m\}$ and $V_{I} \subset \mathbb{C}^{N_{p, q, m}}$ is the subspace of complex dimension $(|I|-1)(p+1)+(m-|I|+1) q$ defined by conditions (a) and (b); here $|I|$ is the cardinality of $I$.

Remark 2.1. All subspaces $V_{I}$ with $|I|=1$ coincide, while all other $V_{I}$ are pairwise distinct; hence, $\mathcal{C}\left(\mathcal{F} ; \mathbb{C}^{N_{p, q, m}}\right)$ is the union of $2^{m}-m$ pairwise distinct subspaces of $\mathbb{C}^{N_{p, q, m}}$.

Let us present other methods related to the subject of the paper describing the center set $\mathcal{C}\left(\mathcal{F} ; \mathbb{C}^{N_{p, q, m}}\right)$.

If $\mathcal{F}_{k}=\left\{f_{\lambda ; k}\right\}$, where $f_{\lambda ; k}:=P_{k} e^{Q_{k}}, 1 \leq k \leq m$, so that $\sum_{k=1}^{m} f_{\lambda ; k}=f_{\lambda}$, then $\lambda \in \mathcal{C}\left(\mathcal{F} ; \mathbb{C}^{N_{p, q, m}}\right)$ if and only if the functions $f_{\lambda ; 1}, \ldots, f_{\lambda ; k}$ are linearly dependent over $\mathbb{C}$, that is, iff the Wronskian determinant $W\left(f_{\lambda ; 1}, \ldots, f_{\lambda ; k}\right)$ is identically zero. According to VP, Lm. 4, Ex. 1], the family $W\left(\mathcal{F}_{1}, \ldots, \mathcal{F}_{m}\right)$ has a form $\left\{S_{\lambda} e^{T_{\lambda}}\right\}_{\lambda \in \mathbb{C}^{N, q, m}}$, where $S_{\lambda}$ and $T_{\lambda}$ are polynomials of degrees at most $m p+\frac{1}{2} m(m-1)(q-1)$ and $q$, respectively. Thus, due to properties (d), (e) and (f) of cyclicity,

$$
c\left(W\left(\mathcal{F}_{1}, \ldots, \mathcal{F}_{m}\right) ; \mu\right) \leq m p+\frac{1}{2} m(m-1)(q-1) \quad \text { for all } \quad \mu \in \mathbb{C}^{N_{p, q, m}} .
$$

Moreover,

$$
\mathcal{C}\left(\mathcal{F} ; \mathbb{C}^{N_{p, q, m}}\right)=\left\{\lambda \in \mathbb{C}^{N_{p, q, m}}: R_{\lambda} \equiv 0\right\},
$$

that is, the center set is defined as the zero set of at most $m p+\frac{1}{2} m(m-1)(q-1)$ polynomials in $\lambda$.

Next, (2.7) and Theorem 1.2 imply that the cyclicity of the family $\mathcal{F}$ of generalized exponential polynomials satisfies the inequality

$$
c(\mathcal{F} ; \mu) \leq m-1+m p+\frac{1}{2} m(m-1)(q-1)\left(=: c_{p, q, m}\right)
$$

For $\mu \notin C\left(\mathcal{F} ; \mathbb{C}^{N_{p, q, m}}\right)$ (i.e., in the case of the multiplicity of zero at $0 \in \mathbb{D}$ of $f_{\mu} \not \equiv 0$ ) this result was proved in $[\mathrm{VP}$. 
Inequality (2.8) and property (c) of cyclicity give an alternative description of the center set of $\mathcal{F}($ cf. $(2.5),(2.6))$ :

$$
\mathcal{C}\left(\mathcal{F} ; \mathbb{C}^{N_{p, q, m}}\right)=\left\{\lambda \in \mathbb{C}^{N_{p, q, m}}: a_{0}(\lambda)=\cdots=a_{c_{p, q, m}}(\lambda)=0\right\} .
$$

Also, for any relatively compact subset $K \Subset \mathbb{C}^{N_{p, q, m}}$, there exists a positive number $r_{K}$ such that each function $f_{\lambda}, \lambda \in K$, has at most $c_{p, q, m}$ zeros in $\mathbb{D}_{r_{K}}$ (counting multiplicities).

Let us show that property $\left(\mathrm{b}^{\prime}\right)$ of cyclicity can be strengthen in our case.

Proposition 2.2. Integral closures in the ring of holomorphic polynomials on $\mathbb{C}^{N_{p, q, m}}$ of polynomial ideals $\mathcal{I}_{c_{p, q, m}}(\mathcal{F})$ generated by $a_{0}, \ldots, a_{c_{p, q, m}}$ and $\mathcal{I}(\mathcal{F})$ generated by all the $a_{k}$ coincide.

Proof. Let $\mathbb{D}^{N_{p, q, m}}$ be the open unit polydisk in $\mathbb{C}^{N_{p, q, m}}$. According to property (b) of cyclicity, there exists a constant $A>0$ such that for all $k>c_{p, q, m}$ and $\lambda \in \mathbb{D}^{N_{p, q, m}}$,

$$
\left|a_{k}(\lambda)\right| \leq A \cdot \sup _{\mathbb{D}^{N_{p, q}, m}}\left|a_{k}\right| \cdot \max _{0 \leq i \leq c_{p, q, m}}\left|a_{i}(\lambda)\right| .
$$

Consider a polynomial map $\psi: \mathbb{C}^{N_{p, q, m}} \rightarrow \mathbb{C}^{N_{p, q, m}}$ which sends coordinates $c_{k i}$ to $c_{k i}^{i+1}$, $0 \leq i \leq p$, and coordinates $d_{k j}$ to $d_{k j}^{j}, 1 \leq j \leq q, 1 \leq k \leq m$. Note that $\psi$ maps $\mathbb{D}^{N_{p, q, m}}$ onto $\mathbb{D}^{N_{p, q, m}}$ and, according to (2.6),

$$
a_{k}(\psi(z \cdot \lambda))=z^{k+1} \cdot a_{k}(\psi(\lambda)) \text { for all } k \in \mathbb{Z}_{+}, z \in \mathbb{C} .
$$

These and (2.9) imply, for all $\lambda \in \mathbb{C}^{N_{p, q, m}}$,

$$
\begin{aligned}
& \left|a_{k}(\psi(\lambda))\right| \leq A \cdot\|\lambda\|_{\infty}^{k+1} \cdot \sup _{\mathbb{D}^{N_{p}, q, m}}\left|a_{k}\right| \cdot \max _{0 \leq i \leq c_{p, q, m}}\left\{\frac{\left|a_{i}(\psi(\lambda))\right|}{\|\lambda\|_{\infty}^{i+1}}\right\} \\
& \leq A \cdot\left(\max \left\{1,\|\lambda\|_{\infty}\right\}\right)^{k} \cdot \sup _{\mathbb{D}^{N_{p, q, m}}}\left|a_{k}\right| \cdot \max _{0 \leq i \leq c_{p, q, m}}\left|a_{i}(\psi(\lambda))\right| ;
\end{aligned}
$$

here $\|\cdot\|_{\infty}$ is $\ell_{\infty}$ norm on $\mathbb{C}^{N_{p, q, m}}$.

Finally, by the definition of the map $\psi$ we have for all $\lambda \in \mathbb{C}^{N_{p, q, m}}$ with $\|\lambda\|_{\infty} \geq 1$,

$$
\|\lambda\|_{\infty}=\left\|\psi^{-1}(\psi(\lambda))\right\|_{\infty} \leq\|\psi(\lambda)\|_{\infty} .
$$

Since $\psi$ is surjective, from the previous two formulas we get, for all $\lambda \in \mathbb{C}^{N_{p, q, m}}$,

$$
\left|a_{k}(\lambda)\right| \leq A \cdot\left(\max \left\{1,\|\lambda\|_{\infty}\right\}\right)^{k} \cdot \sup _{\mathbb{D}^{N, q, m}}\left|a_{k}\right| \cdot \max _{0 \leq i \leq c_{p, q, m}}\left|a_{i}(\lambda)\right| .
$$

These inequalities together with [HRT, Prop. 1.1 (c)] imply that each polynomial $a_{k}$ with $k>c_{p, q, m}$ is integral over the ideal $\mathcal{I}_{c_{p, q, m}}(\mathcal{F})$. This gives the required statement.

Remark 2.3. Proposition 2.2 and the Briançon-Skoda-type theorem, see, e.g., [HS, Cor. 13.3.4], imply that all coefficients of the Maclaurin series expansion of the family $\mathcal{F}^{N_{p, q, m}}=\left\{f_{\lambda}^{N_{p, q, m}}\right\}$ belong to $\mathcal{I}_{c_{p, q, m}}(\mathcal{F})$. An interesting question is whether the ideal $\mathcal{I}_{c_{p, q, m}}(\mathcal{F})$ is integrally closed?

Next, we present the Cartan-type estimates for the family $\mathcal{F}$.

For a nonpolynomial $f_{\lambda}=\sum_{k=1}^{m} P_{k} e^{Q_{k}}$ and $w \in \mathbb{C}$ by $R_{\lambda ; w}$ we denote a (unique) positive number such that

$$
\max _{1 \leq k \leq m} \sup _{\mathbb{D}_{R_{\lambda} ; w}(w)}\left|Q_{k}\right|=1
$$

here $\mathbb{D}_{R}(w):=\{z \in \mathbb{C}:|z-w|<R\}$. 
Proposition 2.4. There is a number $R_{\mathcal{F}}>0$ such that for each nonpolynomial $f_{\lambda} \in \mathcal{F}$ and $R \in\left(0, R_{\lambda ; w} R_{\mathcal{F}}\right)$, and fixed $H \in(0,1]$ and $d>0$ there exists a family of open disks $\left\{D_{j ; \lambda}\right\}_{1 \leq j \leq k}, k \leq c_{p, q, m}$, with $\sum r_{j ; \lambda}^{d} \leq \frac{(2 H R)^{d}}{d}$, where $r_{j ; \lambda}$ is the radius of $D_{j ; \lambda}$, such that

$$
\left|f_{\lambda}(z)\right| \geq \sup _{\mathbb{D}_{R}(w)}\left|f_{\lambda}\right| \cdot\left(\frac{H}{A}\right)^{c_{p, q, m}+1} \quad \text { for all } \quad z \in \mathbb{D}_{R / e}(w) \backslash \cup_{j} D_{j ; \lambda} .
$$

(Here $A=e^{\left(\frac{e+1}{e-1}\right)^{2}}$.)

Proof. Recall that $\lambda=\left(\left(c_{k i}\right)_{0 \leq i \leq p},\left(d_{k j}\right)_{1 \leq j \leq q}\right)_{1 \leq k \leq m}$, where coordinates $c_{k i}$ and $d_{k j}$ are coefficients of polynomials $P_{k}$ and $Q_{k}$, respectively. Consider the compact set

$$
K_{1}:=\left\{\lambda \in \mathbb{C}^{N_{p, q, m}}: \sum_{k, i}\left|c_{k i}\right|=1, \max _{1 \leq k \leq m} \sup _{\mathbb{D}}\left|Q_{k}\right|=1\right\} \Subset \mathbb{C}^{N_{p, q, m}} .
$$

We apply property $\left(\mathrm{h}^{\prime}\right)$ of cyclicity (see (1.3) $)$ to $K_{1}$ and $\varepsilon=1$, and set $R_{\mathcal{F}}:=R_{1}$ (here $R_{\varepsilon}$ is as in property (h)). Then from the corresponding inequality (1.3) using that $A^{-1}\left(\frac{H}{A}\right)^{c(\mathcal{F} ; K)} \geq\left(\frac{H}{A}\right)^{c_{p, q, m}+1}$ we get inequality (2.11) for $w=0, R_{\lambda ; w}=1$. The general case is easily reduced to the previous one by the substitution $z \mapsto R_{\lambda ; w}(z+w), z \in \mathbb{C}$. We leave the details to the readers.

If $f_{\lambda}$ is a polynomial, then its degree is at most $p$ and it satisfies the analog of inequality (2.11) with $c_{p, q, m}+1$ replaced by $p$ for all $R>0$.

Similarly, property $\left(\mathrm{h}^{\prime \prime}\right)$ of cyclicity (see (1.4)) and the arguments of the proof of the previous proposition yield Remez-type inequalities for the family of generalized exponential polynomials.

Proposition 2.5. There exists an absolute constant $\hat{c} \geq 1$ such that for each nonpolynomial $f_{\lambda} \in \mathcal{F}$, an interval $I \subset \mathbb{D}_{\left(R_{\lambda ; w} R_{\mathcal{F}}\right) / e}(w)$ and a measurable subset $\omega \subset I$ the following inequality holds:

$$
\sup _{I}\left|f_{\lambda}\right| \leq \Phi\left(\frac{2 m_{1}(I)}{m_{1}(\omega)}-1\right)^{\hat{c} \cdot \max \left\{1, c_{p, q, m}\right\}} \cdot \sup _{\omega}\left|f_{\lambda}\right| .
$$

If $f_{\lambda}$ is a polynomial, then instead of (2.12) we have the classical Remez inequality with the factor on the right-hand side replaced by $T_{p}\left(\frac{2 m_{1}(I)}{m_{1}(\omega)}-1\right)$, where $T_{p}$ is the Chebyshev polynomial of degree $p$, valid for all measurable $\omega \subset I \Subset \mathbb{C}$.

Remark 2.6. Using inequality (2.12) one obtains various local distributional inequalities for the family of multidimensional generalized exponential polynomials of the form $\sum_{k=1}^{m} P_{k} e^{Q_{k}}$, where $P_{k}$ and $Q_{k}$ are holomorphic polynomials on $\mathbb{C}^{N}$ of degrees $p$ and $q$, respectively (for the corresponding references and results see the Introduction and Section 2 in $[\mathrm{Br} 2]$.

Theorem 1.2 allows to obtain effective estimates for the cyclicity of more complicated families of functions (for instance, the family $\sum_{k=1}^{m} \mathcal{F}_{k 1} e^{\mathcal{F}_{k 2}}$, where all $\mathcal{F}_{k i}$ are families of generalized exponential polynomials). Using then the properties of cyclicity one obtains for such families results similar to those described in the present section.

\section{Proof of Theorem 1.2}

3.1. Equivalent Definition of Cyclicity. For a domain $O \Subset V \subset \mathbb{C}^{n}$ by $\mathcal{O}_{c}(\mathbb{D}, O)$ we denote the set of holomorphic maps $\varphi: \overline{\mathbb{D}} \rightarrow O$ (i.e., each $\varphi$ is holomorphic in a suitable open neighbourhood of the closure $\overline{\mathbb{D}}$ of $\mathbb{D}$.) Let $\mathcal{F}=\left\{f_{\lambda}\right\}_{\lambda \in V}$ be the family of holomorphic functions in the disk $\mathbb{D}_{\rho}$ depending holomorphically on $\lambda \in V$ and $\varphi \in \mathcal{O}_{c}(\mathbb{D}, O)$ be such 
that $\varphi(\mathbb{D}) \not \subset \mathcal{C}(\mathcal{F} ; O)$. We will assume that $\mathcal{F} \neq 0$, i.e., it contains nonidentically zero functions. Then the family $\mathcal{F}_{\varphi}=\left\{f_{\varphi(w)}\right\}_{w \in \mathbb{D} \backslash \varphi^{-1}(\mathcal{C}(\mathcal{F} ; O))}$ consists of nonidentically zero holomorphic functions on $\mathbb{D}_{\rho}$ and its center $\operatorname{set} \mathcal{C}\left(\mathcal{F}_{\varphi} ; \mathbb{D}\right)=\varphi^{-1}(\mathcal{C}(\mathcal{F} ; O))$ consists of finitely many points. Let us consider the Maclaurin series expansion of $\mathcal{F}_{\varphi}$,

$$
f_{\varphi(w)}(z)=\sum_{k=0}^{\infty} c_{k}(w) z^{k}, \quad z \in \mathbb{D}_{\rho}, \quad c_{k} \in \mathcal{O}(\mathbb{D}) .
$$

Let $b_{\varphi}(\mathcal{F}) \in \mathbb{Z}_{+}$be the minimal number such that the ideal $\mathcal{I}\left(\mathcal{F}_{\varphi} ; \mathbb{D}\right) \subset \mathcal{O}(\mathbb{D})$ generated by all the $c_{k}$ coincides with the ideal $\mathcal{I}_{b_{\varphi}(\mathcal{F})}\left(\mathcal{F}_{\varphi} ; \mathbb{D}\right) \subset \mathcal{O}(\mathbb{D})$ generated by $c_{0}, \ldots, c_{b_{\varphi}(\mathcal{F})}$. Then Theorem 1.3 of [Br1] states that for a compact subset $K \subset V$

$$
c(\mathcal{F} ; K)=\lim _{O \rightarrow K} \sup _{\varphi \in \mathcal{O}_{c}(\mathbb{D}, O)} b_{\varphi}(\mathcal{F}),
$$

where the limit is taken over the filter of open neighbourhoods of $K$.

3.2. Proof of Theorem 1.2. According to property (a) of cyclicity, it suffices to prove the result for $K=\{\mu\}$, a point in $V$. Without loss of generality we assume that $\mathcal{F} \neq 0$. Thus, due to (3.13), there exists $\varphi \in \mathcal{O}_{c}(\mathbb{D}, O), \varphi(\mathbb{D}) \not \subset \mathcal{C}(\mathcal{F} ; O)$, where $O$ is an open neighbourhood of $\mu$ such that

$$
c(\mathcal{F} ; \mu)=b_{\varphi}(\mathcal{F}) .
$$

Recall that $\mathcal{F}:=\sum_{j=1}^{k} \mathcal{F}_{j}$, where $\mathcal{F}_{j}:=\left\{f_{\lambda ; j}\right\}_{\lambda \in V}, 1 \leq j \leq k$. By $S_{\lambda} \subset \mathcal{O}\left(\mathbb{D}_{\rho}\right)$ we denote the vector space generated by $f_{\lambda ; 1}, \ldots, f_{\lambda ; k}, \lambda \in V$, and by $d_{\lambda}$ its complex dimension, so that $0 \leq d_{\lambda} \leq k$. We set

$$
d_{\varphi}:=\sup _{w \in \mathbb{D}} d_{\varphi(w)}
$$

By the definition, $d_{\varphi} \geq 1$ and there exist $w_{0} \in \mathbb{D}$ and $1 \leq j_{1}<\cdots<j_{d_{\varphi}} \leq k$ such that $S_{\varphi\left(w_{0}\right)} \subset \mathcal{O}\left(\mathbb{D}_{\rho}\right)$ coincides with the space generated by $f_{\varphi\left(w_{0}\right) ; j_{s}}, 1 \leq s \leq d_{\varphi}$, and $d_{\varphi\left(w_{0}\right)}=d_{\varphi}$

We set

$$
g_{w ; s}:=f_{\varphi(w) ; j_{s}}, \quad W_{w ; p}:=W\left(g_{w ; 1}, \ldots, g_{w ; s}\right), \quad 1 \leq s \leq d_{\varphi}, \quad \text { and } \quad W_{w ; 0}:=1 .
$$

Then the center set of the family $\left\{W_{w ; d_{\varphi}}\right\}_{w \in \mathbb{D}}$ consists of finitely many points and outside these points each $g_{w}:=\sum_{j=1}^{k} f_{\varphi(w) ; j}$ satisfies the ordinary differential equation with meromorphic coefficients (the Frobenius formula, see $[\mathrm{F}]$ ):

$$
\frac{W_{w ; d_{\varphi}}}{W_{w ; d_{\varphi}-1}} \cdot \frac{d}{d z} \cdot \frac{W_{w ; d_{\varphi}-1}^{2}}{W_{w ; d_{\varphi}} \cdot W_{w ; d_{\varphi}-2}} \cdot \frac{d}{d z} \cdots \frac{d}{d z} \cdot \frac{W_{w ; 1}^{2}}{W_{w ; 2} \cdot W_{w ; 0}} \cdot \frac{d}{d z} \cdot \frac{W_{w ; 0}}{W_{w ; 1}} \cdot g_{w}=0
$$

Next, consider the Taylor series expansions of families $\left\{g_{w}\right\}$ and $\left\{W_{w ; s}\right\}, 1 \leq s \leq d_{\varphi}$,

$$
\begin{aligned}
& g_{w}(z)=\sum_{k=0}^{\infty} c_{k}(w) z^{k}, \quad z \in \mathbb{D}_{\rho}, \quad c_{k} \in \mathcal{O}(\mathbb{D}) ; \\
& W_{w ; s}(z)=\sum_{k=0}^{\infty} b_{k ; s}(w) z^{k}, \quad z \in \mathbb{D}_{\rho}, \quad b_{k ; s} \in \mathcal{O}(\mathbb{D}), \quad 1 \leq s \leq d_{\varphi} .
\end{aligned}
$$

By definition, the center sets of these families are subsets of the center set, say $C$, of the family $\left\{W_{w ; d_{\varphi}}\right\}_{w \in \mathbb{D}}$. In particular, they are finite subsets of $\mathbb{D}$. Thus, there exist univariate holomorphic polynomials $P$ and $P_{s}$ with zeros (if any) in $C$, functions $\tilde{c}_{k}$ and $\tilde{b}_{k ; s} \in \mathcal{O}(\mathbb{D})$ and numbers $l_{s} \in \mathbb{Z}_{+}, 1 \leq s \leq d_{\varphi}$, such that $c_{k}=P \cdot \tilde{c}_{k}$ and $b_{k ; s}=P_{s} \cdot \tilde{b}_{k ; s}$ for all $k$ and $s$, and each of the families $\left\{\tilde{c}_{0}, \ldots, \tilde{c}_{b_{\varphi}(\mathcal{F})}\right\}$ and $\left\{\tilde{b}_{0 ; s}, \ldots, \tilde{b}_{l_{s} ; s}\right\}, 1 \leq s \leq d_{\varphi}$, has no common 
zeros on $\mathbb{D}$. (Therefore by the corona theorem, see, e.g., GR, the ideals generated by these families in $\mathcal{O}(\mathbb{D})$ coincide with $\mathcal{O}(\mathbb{D})$.)

We set

$$
\begin{aligned}
& \tilde{g}_{w}(z)=\sum_{k=0}^{\infty} \tilde{c}_{k}(w) z^{k}, \quad z \in \mathbb{D}_{\rho} ; \\
& \widetilde{W}_{w ; s}(z)=\sum_{k=0}^{\infty} \tilde{b}_{k ; s}(w) z^{k}, \quad z \in \mathbb{D}_{\rho}, \quad 1 \leq s \leq d_{\varphi} .
\end{aligned}
$$

Then (3.14) implies

$$
\frac{\widetilde{W}_{w ; d_{\varphi}}}{\widetilde{W}_{w ; d_{\varphi}-1}} \cdot \frac{d}{d z} \cdot \frac{\widetilde{W}_{w ; d_{\varphi}-1}^{2}}{\widetilde{W}_{w ; d_{\varphi}} \cdot \widetilde{W}_{w ; d_{\varphi}-2}} \cdot \frac{d}{d z} \cdots \frac{d}{d z} \cdot \frac{\widetilde{W}_{w ; 1}^{2}}{\widetilde{W}_{w ; 2} \cdot W_{w ; 0}} \cdot \frac{d}{d z} \cdot \frac{W_{w ; 0}}{\widetilde{W}_{w ; 1}} \cdot \tilde{g}_{w}=0 .
$$

Further, by the definition of $b_{\varphi}(\mathcal{F})$, there exists $w_{*} \in \mathbb{D}$ such that $\tilde{c}_{b_{\varphi}(\mathcal{F})}\left(w_{*}\right) \neq 0$ but $\tilde{c}_{k}\left(w^{*}\right)=0$ for all $k<b_{\varphi}(\mathcal{F})(=c(\mathcal{F} ; \mu))$. In what follows, by $m_{s}$ we denote the multiplicity of zero of $\widetilde{W}_{w_{*} ; s}$ at $0 \in \mathbb{D}, 1 \leq s \leq d_{\varphi}$. By the definition of cyclicity (cf. [Br1, Th. 1.3]) $m_{s} \leq c\left(W\left(\mathcal{F}_{j_{1}}, \ldots, \mathcal{F}_{j_{s}}\right) ; \mu\right), 1 \leq s \leq d_{\varphi}$.

We are ready to prove the theorem. Assume, on the contrary, that (cf. the formulation of the theorem)

$$
c(\mathcal{F} ; \mu)>\max _{I \in \mathcal{K}}\left\{c\left(\mathcal{F}_{I} ; \mu\right)+|I|-1\right\} .
$$

We apply the operator on the left-hand side (3.15) with index $w_{*}$ to $\tilde{g}_{w_{*}}$ and compute the resulting multiplicity of zero at $0 \in \mathbb{D}$. By our hypothesis (3.16), the multiplicity of zero at $0 \in \mathbb{D}$ of

$$
\frac{W_{w_{*} ; 0}}{\widetilde{W}_{w_{*} ; 1}} \cdot \tilde{g}_{w_{*}}
$$

is at least $c(\mathcal{F} ; \mu)-m_{1} \geq c(\mathcal{F} ; \mu)-c\left(\mathcal{F}_{j_{1}} ; \mu\right) \geq 1$. Similarly, the multiplicity of zero at $0 \in \mathbb{D}$ of

$$
\frac{\widetilde{W}_{w_{*} ; 1}^{2}}{\widetilde{W}_{w_{*} ; 2} \cdot W_{w_{*} ; 0}} \cdot \frac{d}{d z} \cdot \frac{W_{w_{*} ; 0}}{\widetilde{W}_{w_{*} ; 1}} \cdot \tilde{g}_{w_{*}}
$$

is at least $\left(c(\mathcal{F} ; \mu)-m_{1}-1\right)+\left(2 m_{1}-m_{2}\right)=c(\mathcal{F} ; \mu)+m_{1}-m_{2}-1 \geq c(\mathcal{F} ; \mu)-$ $c\left(W\left(\mathcal{F}_{j_{1}}, \mathcal{F}_{j_{2}}\right) ; \mu\right)-1 \geq 1$, etc. After $d_{\varphi}$ steps we obtain that the multiplicity of zero at $0 \in \mathbb{D}$ of

$$
\frac{\widetilde{W}_{w_{*} ; d_{\varphi}-1}^{2}}{\widetilde{W}_{w_{*} ; d_{\varphi}} \cdot \widetilde{W}_{w_{*} ; d_{\varphi}-2}} \cdot \frac{d}{d z} \cdots \frac{d}{d z} \cdot \frac{\widetilde{W}_{w_{*} ; 1}^{2}}{\widetilde{W}_{w_{*} ; 2} \cdot W_{w_{*} ; 0}} \cdot \frac{d}{d z} \cdot \frac{W_{w_{*} ; 0}}{\widetilde{W}_{w_{*} ; 1}} \cdot \tilde{g}_{w_{*}}
$$

is at least $c(\mathcal{F} ; \mu)+m_{d_{\varphi}-1}-m_{d_{\varphi}}-d_{\varphi}+1 \geq c(\mathcal{F} ; \mu)-c\left(W\left(\mathcal{F}_{j_{1}}, \ldots, \mathcal{F}_{j_{\varphi}}\right) ; \mu\right)-d_{\varphi}+1 \geq 1$. On the other hand, due to (3.15) the function in (3.17) is constant, a contradiction with the previous conclusion.

The proof of the theorem is complete.

\section{REFERENCES}

[B1] N. N. Bautin, Du nombre de cycles limites naissant en cas de variation des coefficients d'un état d'équilibre du type foyer ou centre, C.R. (Doklady) Acad. Sci. URSS (N.S.) 24 (1939), 669-672.

[B2] N. N. Bautin, On the number of limit cycles which appear with the variation of coefficients from an equilibrium position of locus or center type, Transl. AMS, Series I, 5 (1962), 396-413.

[Br1] A. Brudnyi, Cyclicity of zeros of family of analytic functions, GAFA 13 (2003) 1161-1188.

[Br2] A. Brudnyi, On local behavior of holomorphic functions along complex submanifolds of $\mathbb{C}^{N}$, Inventiones Math. 173 (2) (2008), 315-363. 
[F] F. G. Frobenius, Über die Determinante mehrerer Functionen einer Variablen, J. Reine Angew. Math. 77 (1874), 245-257; reprinted in Gesammelte Abhandlungen Band I, 141-153, Springer, Berlin, 1968.

[GR] H. Grauert, R. Remmert, Theorie der Steinschen Räume, Springer-Verlag, New York, 1977.

[HRT] H. Hauser, J.-J. Risler and B. Teissier, The reduced Bautin index of planar vector fields, Duke Math. J. 100 (3) (1999), 425-445.

[HS] C. Huneke and I. Swanson, Integral closure of ideals, rings, and modules, London Math. Soc. Lecture Note Series 336, Cambridge University Press, 2006.

[VP] M. Voorhoeve and A.J. van der Poorten, Wronskian determinants and the zeros of certain functions, Indag. Math. 37 (5) (1975), 417-424.

[Y] Y. Yomdin, Global finiteness properties of analytic families and algebra of their Taylor coefficients, Proc. the Arnoldfest (Toronto, ON, 1997), A.M.S. Providence, RI (1999), 527-555.

Department of Mathematics and Statistics

UNIVERSITY OF CALGARY

Calgary, Alberta

T2N $1 \mathrm{~N} 4$

E-mail address: albru@math.ucalgary.ca 